\title{
Control and Monitoring of Temperature in 3D-Printed Circular Disk Reactors for Continuous Flow Photochemistry using Raspberry Pi Based Software
}

\author{
David Lee Walmsley ${ }^{*}$, Stephen Hilton*, Emilie Sellier†, Matthew Penny, Daniel Maddox† \\ +Vernalis Research, Granta Park, Great Abington, Cambridge, Cambridgeshire CB21 6GB, UK
}

UCL School of Pharmacy, 29-39 Brunswick Square, Bloomsbury, London WC1N 1AX

In this communication we report our investigations into the control and monitoring of temperature in a 3D printed circular disk reactor (CDR) for continuous flow photochemistry using datalogging via a Raspberry Pi microprocessor. A lamp base and holder were designed and realised using 3D printing to enable efficient monitoring of temperature using a temperature probe and the photo-CDR was cooled using a constant controlled flow of compressed air. We demonstrated that temperature gains under commercial LED light sources are significant and that the design of our suitably designed low-cost air-cooled holder could control and stabilise the temperature of the photo-CDR over sustained time-periods.

The control of temperature is a critical factor that contributes to the success or failure of many reactions in synthetic chemistry. Despite this, very little attention has been paid to temperature as a variable in both photocatalytic reactions and reactors with little description of internal temperatures in the literature, leading to difficulty in the reproduction of exact conditions. Svejstrup et al [1] recently described the light intensity and temperature data for a number of commercial photochemistry reactors. They concluded that for the next generation of photoreactors, accurate control of light intensity and reaction temperature would be highly desirable. More recently we described the design and development of a lowcost 3D printed CDR that could be used for continuous flow photochemistry.[2] In that publication we demonstrated that light intensity, as a function of distance from the light source, correlated with conversion using a photocatalysed bromination reaction. In this publication we now wish to describe our recent development in controlling the temperature of the photo-CDR under an LED light source. This was achieved by designing a 3D-printed holder for the CDR which uses compressed air for the cooling and temperature standardisation of the reactor.

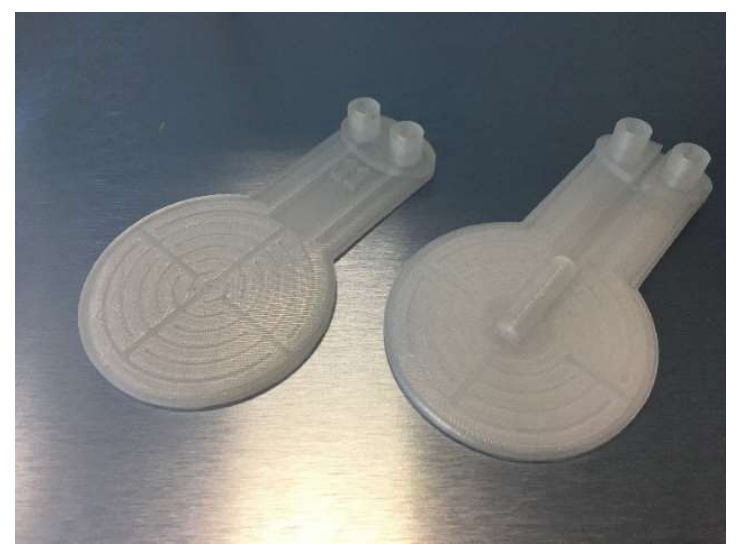

Figure 1. Photo-CDR and Photo-CDR designed with a recess for inserting a temperature probe.

For consistency of temperature measurements, we designed a modified photo-CDR that included a recess in the centre to locate a temperature probe so that we would have a greater record of the temperature of the solution in the photo-CDR. All files for the reproduction of components exemplified in this paper are provided with links to the files in stl format in the supplementary information. Modifications were made to the design of the reflective lamp holder to include a distribution point for a stream of compressed air that was designed to flow both below and over the surface of the reactor and the mirror base of this apparatus in order to maximise the light intensity focused on the photo-CDR and minimise heating from the lamp. Previously we showed that the lowest distance from the lamp and inclusion of a mirror to reflect the light passing through the photo-CDR, led to the highest conversion in our standard reaction. [2] We sought to investigate the temperature that would be observed under these optimum conditions and whether we could lower and control the temperature in order to avoid elevated temperatures leading to undesirable side reactions that would also be applicable to scaleup synthesis. Our desired aim for the air-cooled lamp holder was for it to be amenable to $3 \mathrm{D}$ printing to enable it to be low cost, analogous to the photo-CDR itself. The photo-CDR was printed in polypropylene due to its transparency to blue-light and its chemical resistance.

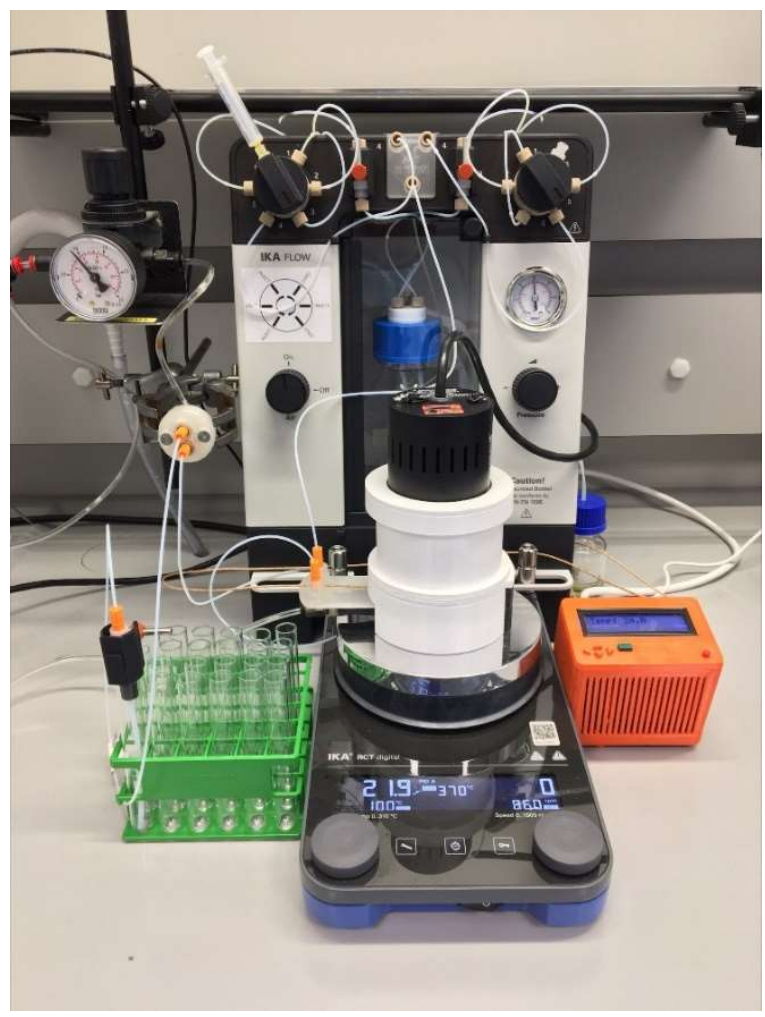

Figure 2. Experimental setup with IKA-Flow system, 3D printed BPR and Raspberry Pi temperature data logger. See Supplementary Information for annotation. 
The non-wetted parts of the cooling housing and lamp holder were printed in the harder material - PLA. One of the critical considerations for additive manufacturing processes such as 3D printing, is the engineering of the printer itself and the limitations of that design and material. Common to many of the lower cost 3D printers, our Ultimaker ${ }^{\mathrm{TM}}$ printer builds 3-dimensional pieces layer by layer onto a bed which is height controlled. The distance from the bed to the print head is increased to build layer upon layer. The consequence of which is that any design has to be built from the bottom upwards. In contrast, this consideration is reversed for SLA printers that print from the top downwards. It is therefore a key consideration in the design of the components, that the base is deliberately designed to be wider than the top.

In order to realise this and minimise support structures, the aircooled lamp holder would need to be assembled from four individual parts; an air-cooled base and the air-cooled lamp housing, which would be assembled from two main parts completed by a capping ring to shroud reflected light (See figure 2).

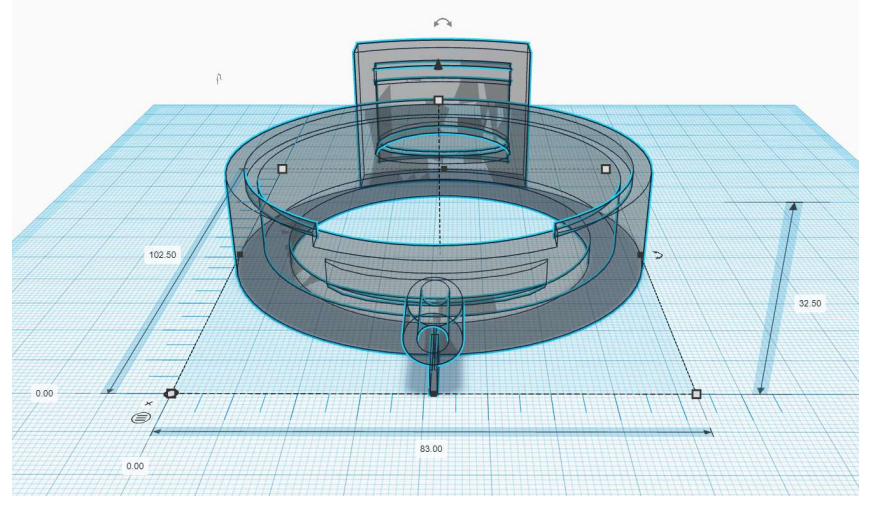

Figure 3. CAD diagram showing the design detail of the air-cooled base.

The dimensions for the lamp holder components were based on the $32 \mathrm{~W}$ Kessil H150W, we also designed an insert that would hold the smaller $18 \mathrm{~W}$ Hepatochem lamp. Having the capacity to use either type interchangeably would subsequently allow us to have access to a wide range of wavelengths subject to the demands of any given photochemistry methods. The air-cooled base (figure 3) was designed to support the photo-CDR above the air entry port and at the opposite end have a conduit to direct the air up and back over the photo-CDR to cool both sides.

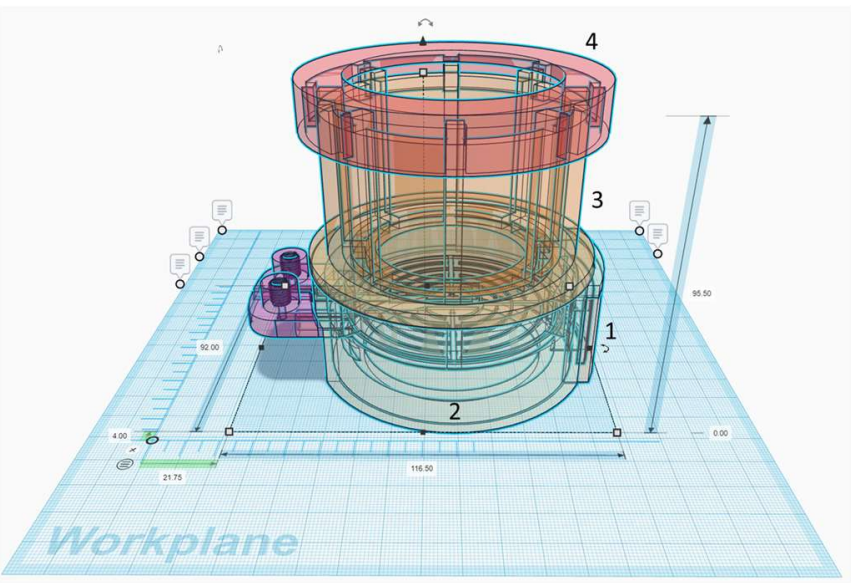

Figure 5. CAD diagram of the assembled air-cooled lamp holder. 1: air-cooled base, 2: lower lamp holder, 3: air cooler upper lamp holder, 4: capping ring. Photo-CDR is shown in situ (purple).
The base was also designed to hold the mirror (70 $\mathrm{mm}$ diameter) which can be readily sourced for low cost. The dimensions were kept to a minimum to reduce waste and print time.

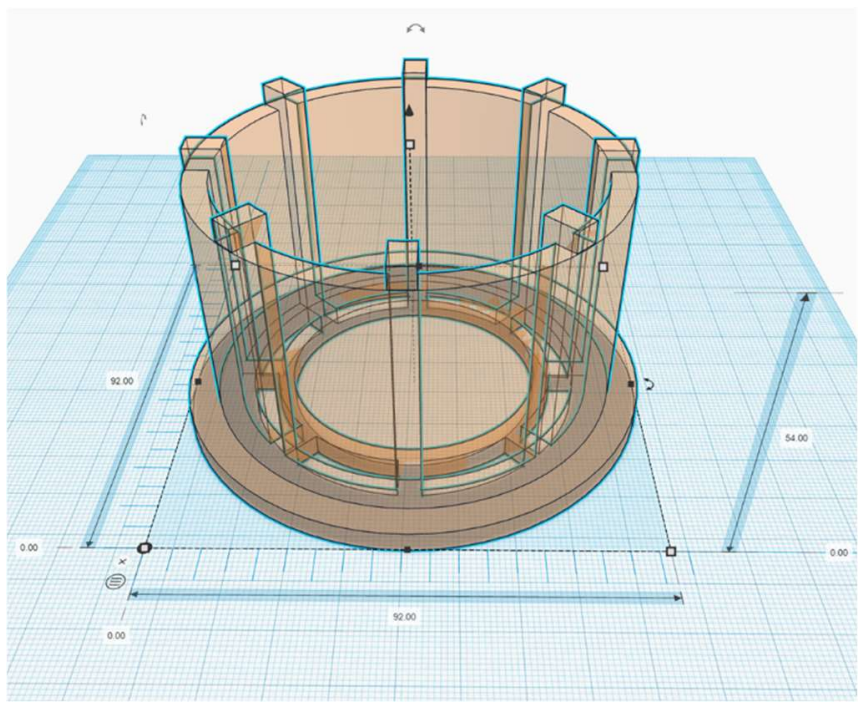

Figure 4. CAD diagram of the upper lamp holder showing design of radial vents.

We designed the lamp holder in 2 parts so that they could be joined. This allowed the lower lamp holder to encase the air-cooled base and direct air up and around the lamp to assist with cooling the lamp itself. Radial vents in the upper lamp holder were intended for this air to vent readily (figure 4). The CAD diagrams for all individual parts are included in the supplementary information.

The mirror was fixed into the cooler base using a polypropylene glue gun and similarly the upper and lower lamp holder were fixed together using polypropylene glue. The assembled cooler base and lamp holder are shown in complete with Kessil H150W $32 \mathrm{~W}$ blue in figure 2 and with Hepatochem $18 \mathrm{~W}$ blue in figure 10

\section{Results and Discussion}

We tested the internal temperature of the photo-CDR by insertion of a temperature probe into the recess of the modified photo-CDR and monitored the temperature over time. We used a temperature probe coupled to a Raspberry $\mathrm{Pi}$ in order to monitor the temperature at the probe every 15 seconds. Details of the temperature probe and script for the Raspberry $\mathrm{Pi}$ are provided in the supplementary information. The light source chosen was the Kessil H150W 32 W $450 \mathrm{~nm}$ (blue). As a control we tested the temperature that would be achieved in the reflective lamp holder that we had used previously for the purpose of shrouding the lamp to prevent excess light escaping. The reflective lamp holder, complete with mirror, was designed to maximise light intensity focused on the photo-CDR. The original design had no cooling aspect to the design, the only cooling coming from advantageous air surrounding the photo-CDR. We stopped collecting data after 10 minutes when $>90^{\circ} \mathrm{C}$ was observed.

Under analogous conditions, without the air turned on, our aircooled lamp holder fared slightly better. Although the maximum temperature stabilised marginally lower at $87{ }^{\circ} \mathrm{C}$, it took significantly longer to reach, $>30$ minutes. This was likely due to the deliberate inclusion of venting around the lamp and above the photo-CDR to allow the air to vent. Once the compressed air was applied from a supply at a pressure of 7 bar, the temperature stabilised at $56^{\circ} \mathrm{C}$ after 13 minutes as shown (Figure 6). 


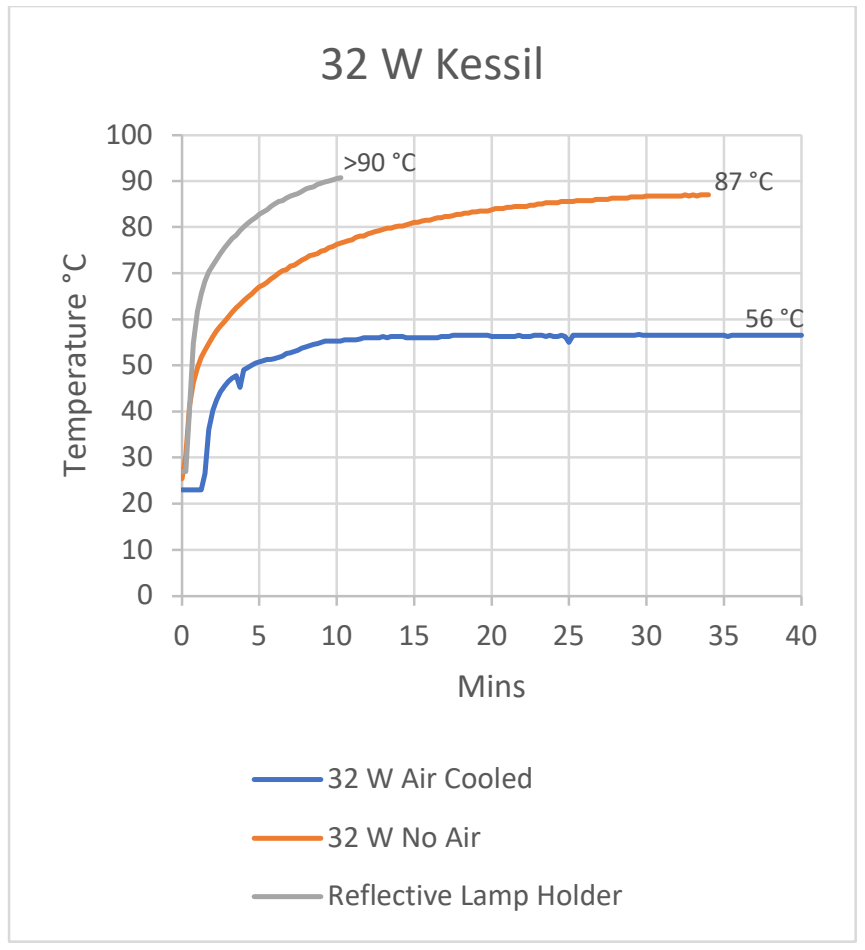

Figure 6. Temperature vs. time in min and labelled with maximum temperature observed.

The effect of air cooling resulted in remarkably stable temperature in the photo-CDR and lowered the temperature by $>30^{\circ} \mathrm{C}$. Ideally any reaction study should be conducted with stability in the temperature and so we planned to switch on the cooling air and the lamp to allow the photo-CDR to stabilise temperature prior to the introduction of the reactants.

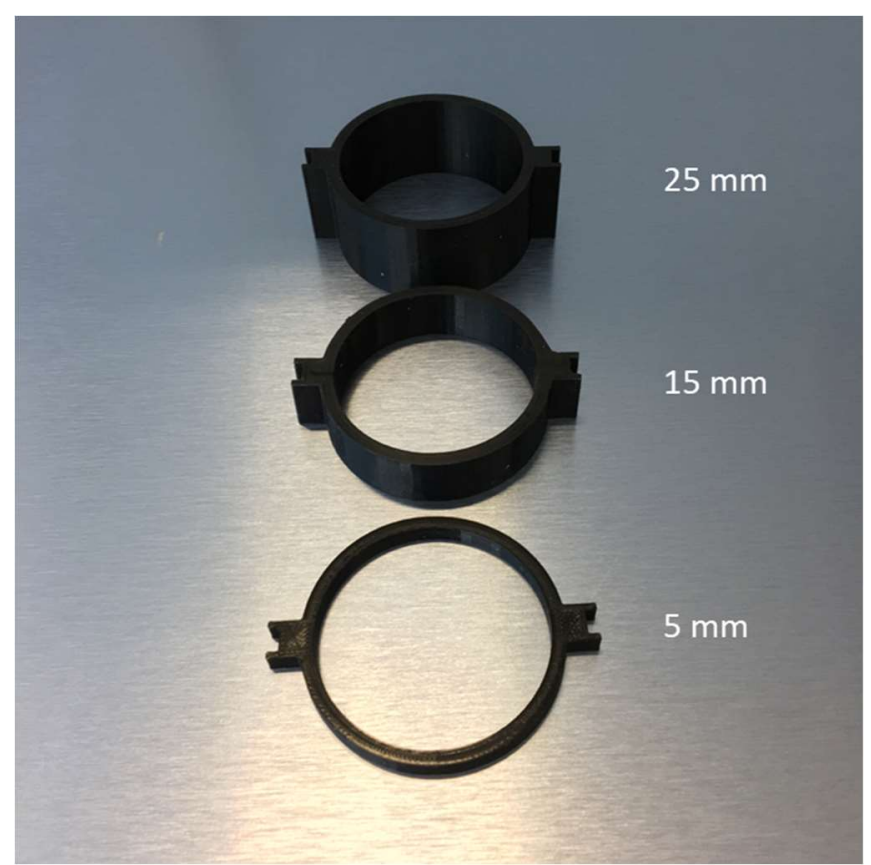

Figure 7. Spacers $5 \mathrm{~mm}, 15 \mathrm{~mm}$, and $25 \mathrm{~mm}$.

We then turned our attention to the effect of light intensity as a function of distance from the photo-CDR. In a previous publication we demonstrated the correlation between distance of the lamp and conversion in a standard bromination reaction.[2] Light intensity decreases as a function of distance according to the Beer-
Lambert Law. Here we studied the temperature over time with the introduction of a spacer between the lamp and photo-CDR (see figure 8 \& 9). The air-cooled lamp holder design had already included $25 \mathrm{~mm}$ space between the surface of the photo-CDR. The spacers were designed at $5 \mathrm{~mm}, 15 \mathrm{~mm}$ and $25 \mathrm{~mm}$ giving an overall distance of the lamp from the photo-CDR of $30 \mathrm{~mm}, 40 \mathrm{~mm}$, and 50 $\mathrm{mm}$ respectively (see figure 7 ). We observed excellent correlation between distance / light intensity and temperature (see figure 8 \& 9). The combination of spacer and the compressed air cooling allowed us to have stable and controlled temperature for a given flow reaction in the photo-CDR reactor.

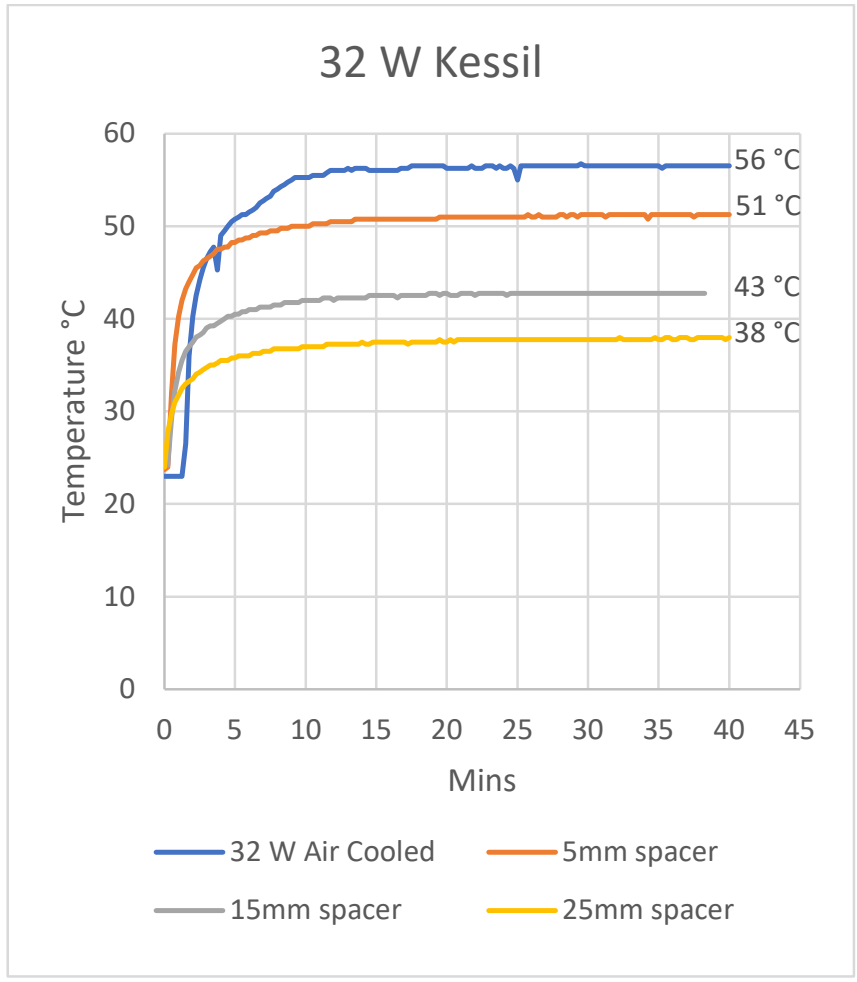

Figure 8 . Temperature vs. time with the inclusion of spacers to increase the distance from the photo-CDR to the $32 \mathrm{~W}$ Kessil lamp.

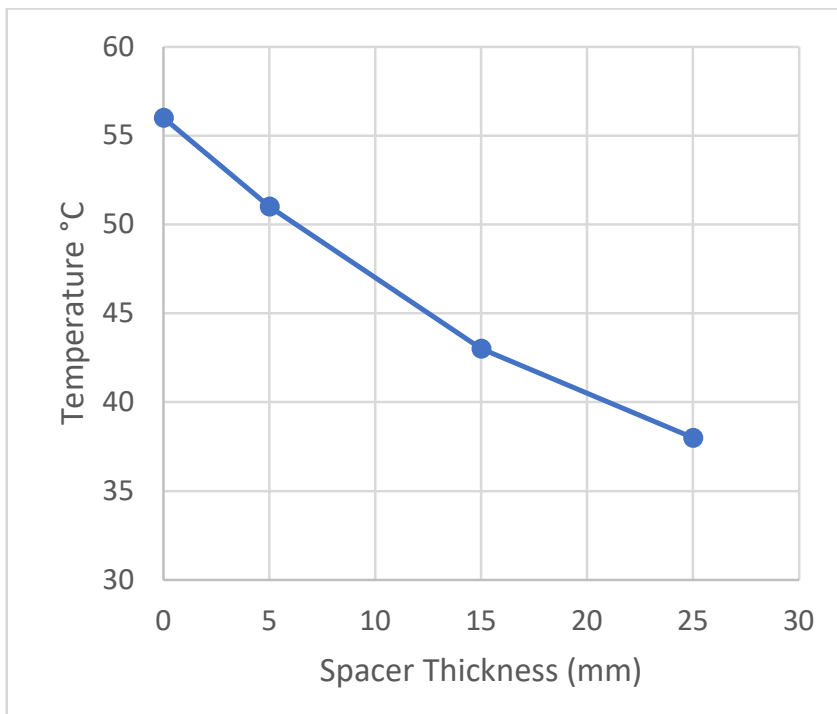

Figure 9. Correlation between size of spacer and maximum temperature. 
We also wanted to investigate light sources from a different vendor to check performance of the air cooler with a different type of LED lamp. A number of lamps are available, and we sourced various wavelength light sources from Hepatochem. For consistency with the data that we obtained from the Kessil lamp, we investigated the $450 \mathrm{~nm} 18 \mathrm{~W}$ lamp from Hepatochem. We needed to insert an adaptor to the lamp holder to hold the smaller Hepatochem lamp securely and the design of this insert is included in the supplementary section. Figure 10 shows the air-cooled lamp holder with the $18 \mathrm{~W}$ Hepatochem $450 \mathrm{~nm}$ lamp.

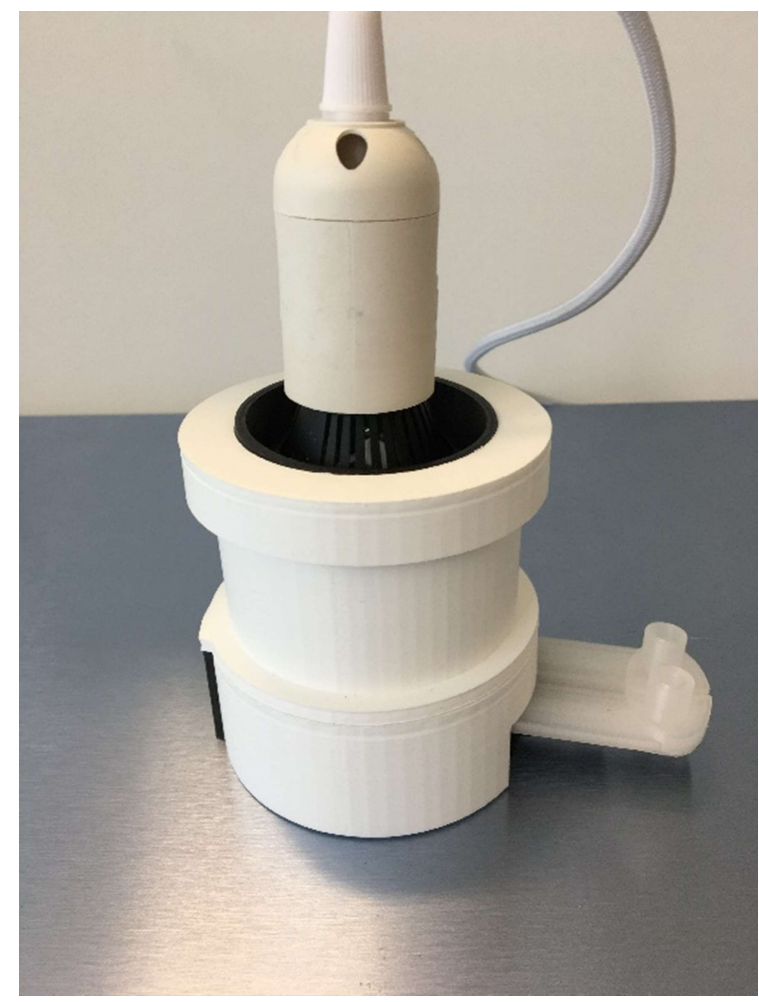

Figure 10. Assembled air-cooled lamp holder with insert to hold the Hepatochem 18 W lamp

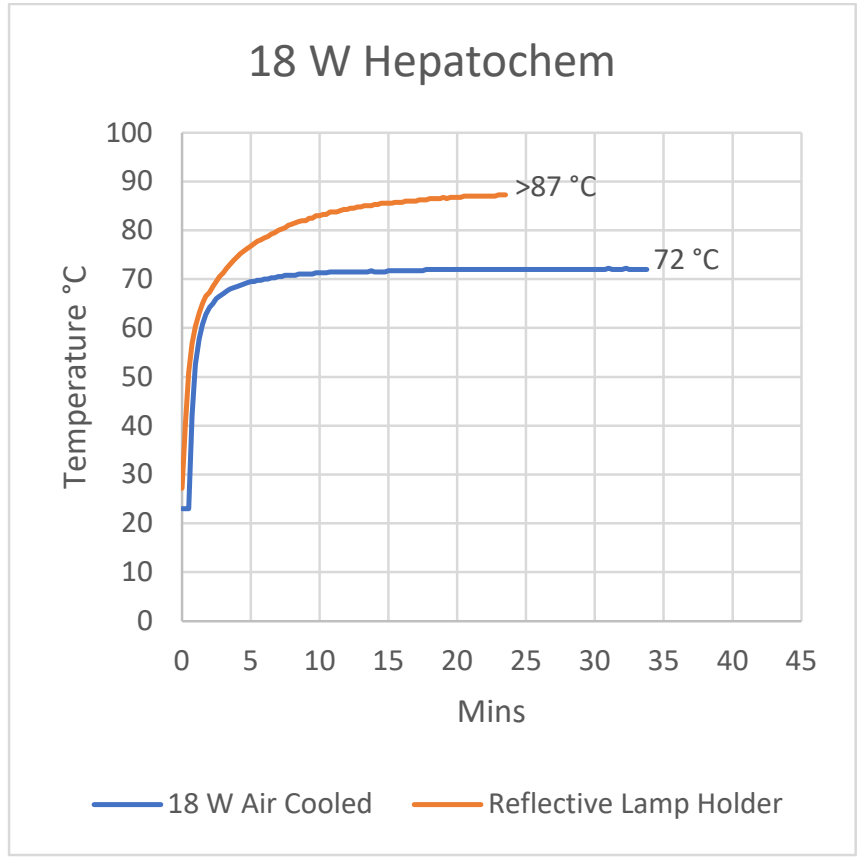

Figure 11. Temperature vs. time using the $18 \mathrm{~W}$ Hepatochem 450 nm lamp.
Using the same conditions and temperature probe insert we studied the temperature profile over time with the Hepatochem lamp.

Counter intuitively the temperature with air cooling stabilised at a higher temperature of $72{ }^{\circ} \mathrm{C}$ in testing using the lower powered Hepatochem lamp. The air surrounding the smaller Hepatochem lamp is able to vent more readily than around the larger Kessil lamp, so we did not find a solution to using the air flow to lower the temperature further (figure 11). The difference in operating temperature of the two types of lamps could be due to a more efficient heat sink incorporated into the $32 \mathrm{~W}$ Kessil lamp.

As in the previous publication, we selected a simple benzylic bromination reaction to act as a standard for comparison [1] (Scheme 1). The $450 \mathrm{~nm}$ blue LED lamps were compared at full power, held $25 \mathrm{~mm}$ above the photo-CDR reactor and compressed air cooling applied to stabilise the temperature of the photo-CDR before introduction of reactants.<smiles>COC(=O)CCc1ccccc1</smiles>
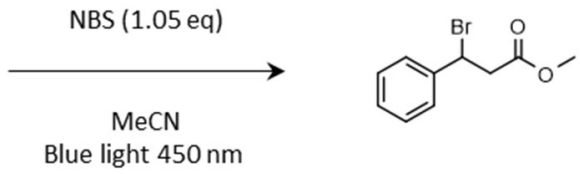

Scheme 1. Selected radical bromination photochemistry reaction for photoCDR testing.

A $0.5 \mathrm{M}$ acetonitrile solution of methyl hydrocinnamate and NBS was then passed through the photo-CDR at $0.5 \mathrm{~mL} / \mathrm{min}$, residence time of 420 seconds, and the degree of conversion to the brominated product determined by NMR (Table 1 ).

Table 1 - Results

\begin{tabular}{ccccc} 
Entry & $\begin{array}{c}\text { light source } \\
(450 \mathrm{~nm})\end{array}$ & $\begin{array}{c}\text { Power } \\
(\mathrm{W})\end{array}$ & $\mathrm{T}\left({ }^{\circ} \mathrm{C}\right)$ & Conv.(\%) \\
\hline 1 & Kessil PR150 & 32 & 58 & 74 \\
2 & Hepatochem & 18 & 65 & 24
\end{tabular}

For the fluidic system we used the IKA-Flow system and controlled the flow rate using either a restrictor or a 3D-printed back-pressure regulator [3]. See supplementary information.

We observed a strong correlation between conversion and the power of the lamp. The higher conversion being observed for the $32 \mathrm{~W}$ lamp vs. the lower power $18 \mathrm{~W}$. Conversion rate was not closely correlated with temperature as we again observed a higher temperature of the photo-CDR reactor with the lower powered lamp and yet saw less conversion to brominated product. Future work could involve an investigation into the lux at the photo-CDR for different lamps. Clearly this would involve adapting the lamp holder to be able include a suitable type of illuminance meter (lux meter) and this is beyond the scope of this paper. In this paper we focused solely on the temperature profile of $450 \mathrm{~nm}$ blue lights that were relevant to the chemistry that we were performing at the time. A further study calibrating the air-cooler for lamps of different wavelengths would extend the scope of the reaction chemistry whilst maintaining control of temperatures for each given wavelength. Again, an investigation into a range of wavelengths is beyond the scope of this paper. 


\section{Conclusion}

We designed an air-cooled lamp holder for the photo-CDR reactors and designed all parts so that they could be obtained at low cost with access to a 3D printer. The design was based around the Kessil H150W 32 W lamp and we made a small insert adapter that could hold the Hepatochem lamp. Reflective surfaces in the cavity and a mirror incorporated into the base unit maximised the light intensity at the photo-CDR. The air-cooling, using a laboratory supply of compressed air, stabilised the temperature at $56{ }^{\circ} \mathrm{C}$. We saw a reduction of $>30{ }^{\circ} \mathrm{C}$ when compared to no compressed air, with lamp in close proximity to the photo-CDR $(25 \mathrm{~mm}$ above the surface). We increased the distance of the lamp above the photoCDR and showed and excellent correlation between distance and temperature with a maximum total distance of $50 \mathrm{~mm}$ in combination with air-cooling lowering the temperature below $40^{\circ} \mathrm{C}$. These findings were consistent with lowering the light intensity, in accordance with the Beer-Lambert law, resulting in lower temperatures. To investigate the lux at the photo-CDR would require further investigation however, we have established that we have excellent stabilisation of temperature using air-cooling. In addition, with a combination of air-cooling and distance using spacers, we were able to have control of a range of fixed temperatures and proportional light intensities. Control over both these parameters are essential to the optimisation of any given photocatalysed reaction. In combination with the air-cooler the photo-CDR allows additional control over other parameters that govern reaction kinetics by virtue of being a flow chemistry reactor.

References

1. Effects of Light Intensity and Reaction Temperature on Photoreactions in Commercial Photoreactors Thomas D. Svejstrup, Anamitra Chatterjee, Denis Schekin, Thomas Wagner, Julia Zach, Magnus J. Johansson, Giulia Bergonzini, Burkhard König https://chemistry-

europe.onlinelibrary.wiley.com/doi/epdf/10.1002/ cptc. 202100059

2. Penny M, Hilton S. 3D Printed Reactors and Kessil Lamp Holders for Flow Photochemistry: Design and System Standardization. ChemRxiv. Cambridge: Cambridge Open Engage; 2021; https://doi.org/10.33774/chemrxiv-2021-kmxgd

This content is a preprint and has not been peerreviewed.

3. Walmsley, L., \& Sellier, E. (2020, January 24). Design and development of a 3D-printed backpressure regulator. https://doi.org/10.31224/osf.io/3t9bq
[*] Corresponding Authors

David Lee Walmsley

Vernalis Research, Granta Park, Great Abington,

Cambridge. CB21 6GB

Email : I.walmsley@vernalis.com

Stephen Hilton

UCL School of Pharmacy

29-39 Brunswick Square, Bloomsbury,

London WC1N 1AX

Email: s.hilton@ucl.ac.uk 